\title{
Triptolide reverses epithelial-mesenchymal transition in glioma cells via inducing autophagy
}

\author{
Minfang Lai ${ }^{1}$, Lili Liu ${ }^{1}$, Long Zhu ${ }^{1}$, Wenping Feng ${ }^{2}$, Jilai Luo ${ }^{2}$, Yawei Liu ${ }^{3}$, Shengze Deng ${ }^{2} \wedge$ \\ ${ }^{1}$ Department of Pharmacy, The First Affiliated Hospital of Nanchang University, Nanchang University, Nanchang, China; ${ }^{2}$ Department of \\ Neurosurgery, The First Affiliated Hospital of Nanchang University, Nanchang University, Nanchang, China; ${ }^{3}$ Laboratory for Precision \\ Neurosurgery, Nanfang Hospital, Southern Medical University, Guangzhou, China \\ Contributions: (I) Conception and design: M Lai, S Deng; (II) Administrative support: Y Liu; (III) Provision of study materials or patients: L Liu, L \\ Zhu; (IV) Collection and assembly of data: W Feng, J Luo; (V) Data analysis and interpretation: M Lai; (VI) Manuscript writing: All authors; (VII) \\ Final approval of manuscript: All authors. \\ Correspondence to: Shengze Deng. Department of Neurosurgery, The First Affiliated Hospital of Nanchang University, Nanchang University, No. 17 \\ Yongwai Zheng Street, Nanchang 330006, China. Email: dengsz03@126.com; Yawei Liu. Laboratory for Precision Neurosurgery, Nanfang Hospital, \\ Southern Medical University, Guangzhou 510515, China. Email: liuyawei@smu.edu.cn.
}

Background: To observe the effects of triptolide (TP) on the proliferation, migration, invasion, and epithelial-mesenchymal transition (EMT) of glioma cells, and to explore the possible mechanisms of phenotypic changes in EMT.

Methods: The U87 and U251 glioma cell lines were treated TP. The Cell Counting Kit-8 (CCK-8) method was used to detect the half-maximal inhibitory concentration $\left(\mathrm{IC}_{50}\right)$ of $\mathrm{TP}$ in these two cell lines and the inhibition of cell proliferation at the $\mathrm{IC}_{50}$ concentration. The wound-healing experiment and Transwell invasion assay were used to detect the cells' migration and invasion abilities, respectively. Using western blot protocol, the expression levels of the EMT markers were analyzed, and the levels of the autophagy markers were also detected. The pEGFP-C2-LC3B plasmid was transfected into glioma cells, and the effect of TP on autophagy was detected by immunofluorescence. A subcutaneous tumor model in nude mice was established to observe the effect of TP on cell proliferation in vivo, and immunohistochemistry (IHC) was used to detect the expression levels of EMT markers in mouse tumor tissues.

Results: TP significantly inhibited the proliferation of U87 and U251 cells in a dose- and time-dependent manner. TP had a significant inhibitory effect on the migration and invasion of U87 and U251 cells. Western blot showed that TP reversed the process of EMT in glioma cells, which was evidenced by the upregulated expression of the epithelial marker E-cadherin, and the downregulated expression of the mesenchymal markers N-cadherin, Vimentin, ZEB1, Snail, and Slug. TP increased autophagy in glioma cells, increased the LC3B II/I ratio, and upregulated Beclin-1 and Atg-7 expression. Immunofluorescence showed that the number of autophagosomes increased significantly after TP was applied to cells. In the nude mouse subcutaneous tumor model, experiments revealed an inhibitory effect of TP on glioma cell proliferation in vivo. IHC confirmed that the expression of E-cadherin was upregulated in mouse tumor tissues, while the expression levels of N-Cadherin and Vimentin were downregulated.

Conclusions: TP can inhibit glioma cell proliferation, migration, and invasion, and reverse EMT progression. The possible mechanism of EMT reversal in glioma cells is that TP induces autophagy.

Keywords: Triptolide (TP); glioma cells; epithelial-mesenchymal transition (EMT); migration and invasion; autophagy

$\wedge$ ORCID: 0000-0001-9600-4222. 
Submitted May 23, 2021. Accepted for publication Aug 11, 2021.

doi: 10.21037/atm-21-2944

View this article at: https://dx.doi.org/10.21037/atm-21-2944

\section{Introduction}

Gliomas are one of the most common malignant intracranial tumors. In 2007, the World Health Organization (WHO) Classification of Tumors of the Central Nervous System divided gliomas into four grades (WHO I-IV) based on their aggressiveness, anaplasticity, and differentiation (1). Glioblastomas (GBMs), which account for almost $50 \%$ of all gliomas, are the most malignant glioma (WHO IV). Although a comprehensive treatment plan (also called the Stupp scheme, which includes surgery, radiotherapy, and chemotherapy) is generally used to treat GBMs in clinical practice, the prognosis of this disease is still extremely poor, with an average median survival time of only approximately 14.6 months (2). One of the reasons for the poor prognosis of $\mathrm{GBM}$ is its resistance to radiotherapy and chemotherapy, and especially to temozolomide (TMZ), which is currently the first-line chemotherapeutic drug used to treat the disease. Many studies have continued to focus on the mechanisms of TMZ resistance $(3,4)$. Another reason is that surgery cannot completely remove the tumor, and even a single tumor cell can invade the surrounding brain parenchyma. In $90 \%$ of patients with glioma, remnant tumor cells are found at the edge of the tumor cavity or within a few centimeters around it (5). Therefore, studying the specific molecular mechanisms of glioma migration and invasion, and applying new drugs or molecular targeted therapies for these mechanisms could improve the prognosis of patients with GBM.

Triptolide (TP) is a purified diterpenoid isolated from the Chinese herbal medicine Tripterygium wilfordii Hook F. TP is a small-molecule (MW 360) fat-soluble drug that can pass easily through the blood-brain barrier (6). It has various biological activities, including immune regulation, anti-proliferation, pro-apoptosis, and anti-inflammatory effects (7). Moreover, an increasing number of preclinical studies have shown that TP has strong antitumor effects. Therefore, TP is an ideal drug candidate for the treatment of glioma.

Epithelial-mesenchymal transition (EMT) refers to the process in which cells lose their polarity, cell-cell junction, and undergo phenotypic changes. Through this process, unipolar, immobile epithelial cells transform into mobile mesenchymal cells. This transformation of cells plays an important role in tumor invasion and metastasis. EMT has been found to be involved in tumor invasion and metastasis in a variety of epithelial tumors, including colon cancer, pancreatic cancer, and breast cancer $(8,9)$. Recently, some scholars also studied the phenotype of EMT in glioma and found that, as the activators of EMT, ZEB1 and ZEB2 of the SNAIL family can, to varying degrees, promote the invasion of GBM in vivo and in vitro $(10,11)$. TP causes changes in EMT-like phenotypes that are closely related to tumor invasion and metastasis, as reported in pancreatic cancer $(12,13)$, lung cancer $(14,15)$, and colon cancer (16). However, whether or not TP causes changes in the EMT of glioma cells is still unknown.

Autophagy is the process of self-digestion of senescent or apoptotic cells and the maintenance of cell energy metabolism, and it plays a key role in maintaining intracellular environmental stability and self-survival in the face of microenvironmental pressure (17). On the one hand, autophagy improves the adaptability of cancer cells to resist apoptosis under pressure; on the other hand, it reduces metastasis by limiting tumor necrosis and preventing inflammatory immune cell infiltration. The physiological role played by autophagy mainly depends on the tumor type and stage (18). Recent studies have shown that autophagy and EMT are both important but distinct links in the process of tumorigenesis and tumor development. In the early stage of tumor development, autophagy inhibits tumor invasion and metastasis by selectively destroying key signaling molecules in the EMT process (19-21). Conversely, during the period of metastasis and diffusion, cells undergoing EMT require activation by autophagy in order to obtain more energy to maintain their survival.

In this study, we aimed to discover the effects of TP on the proliferation, migration, invasion, and EMT of glioma cells, and to explore the possible mechanisms of phenotypic changes in EMT. Different from other previous studies, we had innovatively focused on and discovered the potential relationship between EMT and autophagy in glioma cells under the influence of TP. We present the following article in accordance with the ARRIVE reporting checklist (available at https://dx.doi.org/10.21037/atm-21-2944). 


\section{Methods}

\section{Reagents and antibodies}

The human glioma cell lines U87 and U251 were purchased from the American Type Culture Collection (ATCC; Rockville, MD, USA) and maintained in Dulbecco's Modified Eagle Medium (DMEM glucose $4.5 \mathrm{~g} / \mathrm{L}$; Gibco, Carlsbad, CA, USA) supplemented with $10 \%$ fetal bovine serum (FBS; Gibco) at $37{ }^{\circ} \mathrm{C}$ in a humidified incubator with $5 \% \mathrm{CO}_{2}$. TP (purity >98\%) was purchased from Zelang Biotechnology Co., Ltd. (Nanjing, China), and dimethyl sulfoxide (DMSO) was purchased from Sigma Company in the United States. The stock solutions $(55 \mathrm{~mol} / \mathrm{L})$ were prepared with DMSO and stored in the dark at $-80^{\circ} \mathrm{C}$.

EMT antibody (Sampler Kit \#9782) and autophagy antibody (Sampler Kit \#4445) were obtained from Cell Signaling Technology (Danvers, MA, USA). GAPDH antibody was purchased from ABclonal Technology (Woburn, MA, USA).

\section{Cell viability assay}

Cells were seeded in 96-well plates at a density of $1.5 \times 10^{4}$ cells/well and treated with various concentrations of $\mathrm{TP}(0,1,5,10,20,50,100,200$, or $400 \mathrm{ng} / \mathrm{mL})$ for 48 hours. Cell viability was assessed by performing a Cell Counting Kit-8 (CCK-8) assay (Dojindo, CK04, Japan) following the protocol supplied by the manufacturer. Briefly, after the culture medium had been discarded, an equal amount of CCK-8 reagent was added to each well, before incubation at $37^{\circ} \mathrm{C}$ for 1 hour in the dark. Afterward, the optical density (OD) value at $450 \mathrm{~nm}$ was measured. The cell viability of TP-treated cells was normalized to that of the controls. The half-maximal inhibitory concentration $\left(\mathrm{IC}_{50}\right)$ of $\mathrm{TP}$ was calculated in the experimental groups of cells. Then, the two cell lines were stimulated under the corresponding $\mathrm{IC}_{50}$ concentration of TP.

TP-treated cells were plated at a density of 1,000 cells/well in 96-well plates. After 12-hour incubation to allow cells to attach to the bottom of the well, $10 \mu \mathrm{L}$ CCK- 8 solution was added to each well on days $0,1,2,3,4,5,6$, and 7 , and the plates were incubated for another 1 hours on each day. A microplate reader was then used to determine the $\mathrm{OD}$ value at $450 \mathrm{~nm}$.

\section{Wound-bealing assay}

Confluent cell monolayers were inoculated in 6-well plates and gently scratched with the tip of a $20-\mu \mathrm{L}$ pipette to create uniform, cell-free wounds. Fresh or conditioned medium with or without TP was added. At $0,12,24$, and 48 hours, photomicrographs of the wounds were taken under an inverted microscope (Olympus Corp., Japan). The wound areas were then quantified using ImageJ software.

\section{Cell migration assay}

Cell invasion ability was examined using a Transwell system (product no. 3422; Corning, Inc., USA) with $8.0-\mu \mathrm{m}-$ diameter pores. BD Matrigel was taken out of a $-80{ }^{\circ} \mathrm{C}$ refrigerator and placed in a $4{ }^{\circ} \mathrm{C}$ refrigerator overnight to thaw. Then, $60 \mu \mathrm{L}$ of Matrigel was mixed well with $300 \mu \mathrm{L}$ of serum-free medium at $4^{\circ} \mathrm{C}$. After that, $100 \mu \mathrm{L}$ of mixed solution was added to each upper chamber and incubated at $37^{\circ} \mathrm{C}$ for $4-5$ hours. Control or TP-treated cells $\left(1 \times 10^{4}\right)$ were added to the top chambers and $500 \mu \mathrm{L}$ of conditioned medium containing $20 \%$ FBS was added to the lower chamber. After 24 hours, the cells on the top surface of the inserts were removed with cotton swabs. Cells adhering to the lower surface were fixed with $5 \%$ glutaraldehyde, stained with $0.1 \%$ crystal violet solution, and counted under a microscope across 9 high-resolution predetermined fields. All assays were independently repeated at least 3 times.

\section{Western blot assay}

Cells were lysed in RIPA buffer and the lysates were normalized using a BCA protein assay kit (Beyotime, China). Total proteins were separated by $10-15 \%$ sodium dodecyl sulfate-polyacrylamide gel electrophoresis and transferred to a polyvinylidene fluoride (PVDF) membrane. The membrane was blocked in $5 \%$ non-fat milk and incubated with the indicated primary antibodies at $4{ }^{\circ} \mathrm{C}$ overnight. Then, proteins were detected by incubation with species-specific, peroxidase-conjugated secondary antibodies. The immunoreactive bands were detected using a chemiluminescence kit (Millipore, Plano, TX, USA) and visualized with a KODAK imaging system.

\section{Nude mouse xenografts}

Twelve male BALB/C-nu/nu nude mice were purchased from the Experimental Animal Center of Southern Medical University. The mice were 4-6 weeks old and each weighed about $20 \mathrm{~g}$. U87 cells $\left(1 \times 10^{6}\right)$ in the logarithmic growth phase were injected subcutaneously into the inguinal region 
of 4-week-old male nude mice. After 7 days, the tumors had grown to 3-4 $\mathrm{mm}$ in diameter, and the mice were randomly divided into 2 groups, with 6 mice in each group. Every other day, the mice in 1 group were treated with vehicle (DMSO) and those in the other group were treated with TP solution at $0.5 \mathrm{mg} / \mathrm{kg}$. The mice were sacrificed at day 28 after inoculation, and the tumor tissues were removed and photographed. The tumor volume was calculated using the formula $V=(W / 2)^{2} \times L$, in which $L$ represents tumor length and $\mathrm{W}$ represents tumor width. The expression of key EMT proteins was detected by immunohistochemistry (IHC). This study was performed under a project license (No. 2020117) granted by the ethics board of the First Affiliated Hospital of Nanchang University and was carried out in strict accordance with the recommendations in the Guide for the Care and Use of Laboratory Animals of the National Institutes of Health. The protocol was prepared before the study without registration.

\section{IHC}

To study the expression of key EMT proteins in tumor tissues from the nude mice, IHC was performed following the two-step plus poly-horseradish peroxidase (HRP) method. Briefly, 4- $\mu \mathrm{m}$ tissue sections were mounted on aminopropylethoxysilane slides. The slides were deparaffinized, rehydrated, immersed in $10 \mathrm{mM}$ sodium citrate buffer $(\mathrm{pH} 6.0)$, pretreated in a microwave oven for 20 minutes, and then rinsed for 15 minutes with phosphate-buffered saline (PBS). Endogenous peroxidase was quenched through incubation of the sections in $0.3 \%$ hydrogen peroxide for 30 minutes at room temperature. Non-specific binding was blocked by incubation with nonimmune serum ( $1 \%$ bovine serum albumin for 15 minutes at room temperature). The sections were then incubated overnight with primary antibodies against E-cadherin, $\mathrm{N}$-cadherin, and Vimentin (Sampler Kit \#9782; Cell Signaling Technology, MA, USA) at a dilution of 1:100. The next day, the slides were stained for E-cadherin, $\mathrm{N}$-cadherin, and Vimentin using the two-step plus PolyHRP Anti-Rabbit IgG Detection System (PV-6001; ZSGBBio, Beijing, China). After visualization of the reaction with 3,3'-diaminobenzidine, the slides were counterstained with hematoxylin and mounted with a synthetic medium.

\section{Immunofluorescence staining}

U87 and U251 cells were seeded into a laser confocal dish, at a density with 4-6 cells visible in each high-powered field of the microscope. The constructed pEGFP-C2-LC3B plasmid was transfected into cells using Lipofectamine 2000. After 48 hours, TP was added to the experimental cell group (the concentration was the $\mathrm{IC}_{50}$ of U87 and $\mathrm{U} 251$ cells), and the corresponding volume of DMSO was added to the control group. Six hours later, the cells were rinsed twice with PBS, fixed for 5-10 minutes with 4\% paraformaldehyde, and permeabilized with $0.25 \%$ Triton $\mathrm{X}-100$ for 5 minutes. After fixation and rinsing with PBS, $1 \mathrm{~mL}$ of DAPI (4',6-diamidino-2-phenylindole) was added and the cells were left to stain for 5 minutes. After the DAPI was aspirated, the cells were washed 3 times with PBS, and finally, $100 \mu \mathrm{L}$ PBS was added. Photographs were taken under a confocal laser microscope (LSM880, Carl Zeiss, Germany).

\section{Image, data processing and Statistical analysis}

Using Image J software, the wound-healing assay results were analyzed by calculating the scratch areas of different groups of cells at different time points and dividing them by the initial scratch area to obtain the scratch area ratio. For the western blot experiment, the relative expression level of the protein in each target band was obtained by dividing the gray value of GAPDH (as the internal reference), also using Image J.

GraphPad Prism 5.0 software was used for data processing and chart drawing, and SPSS 23.0 software was used for statistical analysis. All data in this study were expressed as the means \pm standard deviations (SDs) from at least 3 independent experiments. Comparisons between 2 groups were drawn by using the Student's $t$ test. Statistical significance was indicated by $\mathrm{P}<0.05$ or $\mathrm{P}<0.01$.

\section{Results}

\section{TP inbibits the proliferation of glioma cells in vitro and in vivo}

At 48 hours, the $\mathrm{IC}_{50}$ of TP in the U87 and $\mathrm{U} 251$ cell lines was $20 \mathrm{ng} / \mathrm{mL}$ and $50 \mu \mathrm{g} / \mathrm{mL}$, respectively. Then, both cell lines were stimulated with the corresponding $\mathrm{IC}_{50}$ concentration of $\mathrm{TP}$, and the cell proliferation activity was detected using the CCK- 8 method. From day 2 after treatment, the cell proliferation activity of TP-treated cells began to decrease compared to that of the control group, and the difference became increasingly significant as time 

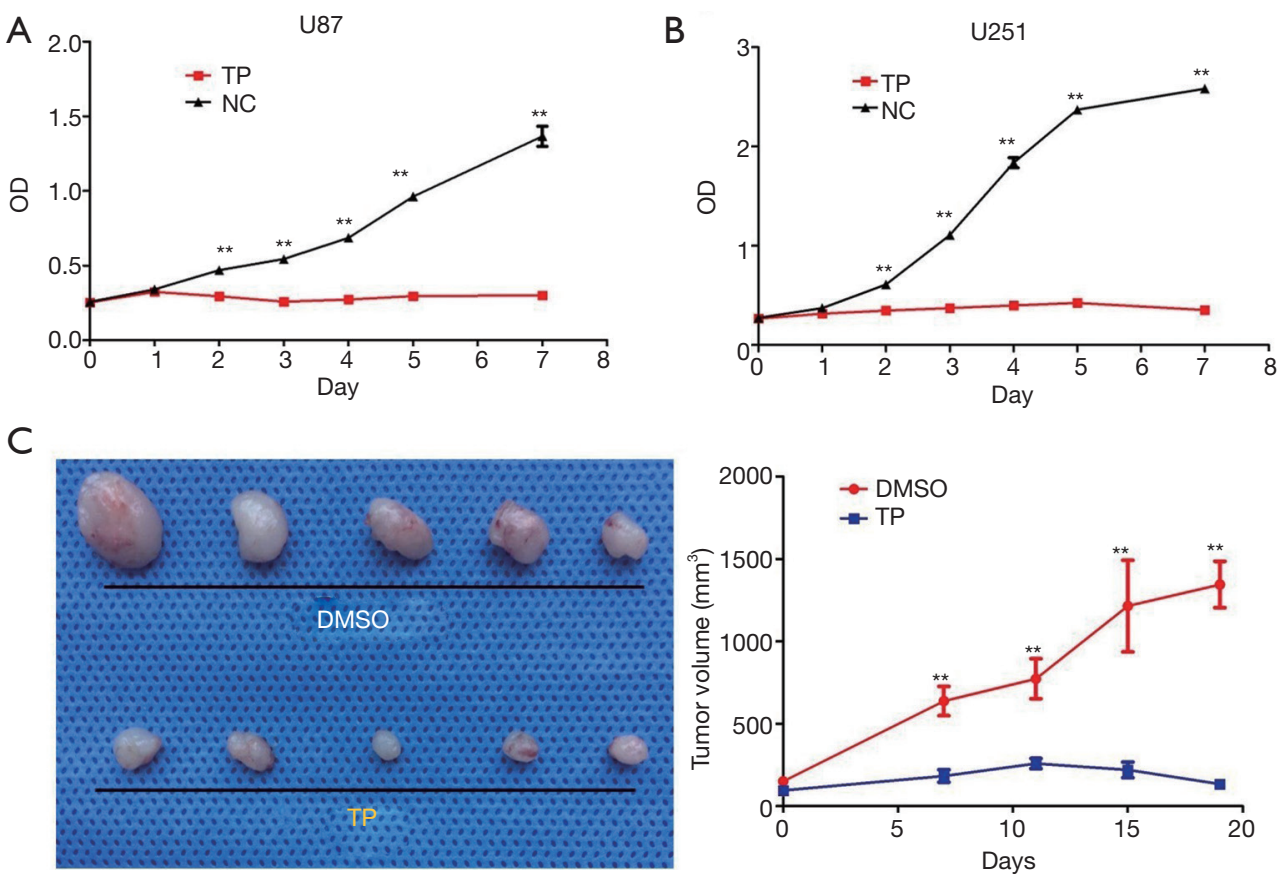

Figure 1 Effects of TP on the proliferative activity of glioma cells in vitro and in vivo. (A) The changes in U87 cell proliferation after 7 days of TP treatment at the $\mathrm{IC}_{50}$ concentration $(20 \mathrm{ng} / \mathrm{mL})$, as determined by the CCK-8 method. (B) The changes in U251 cell proliferation after 7 days of TP treatment at the $\mathrm{IC}_{50}$ concentration $(50 \mathrm{ng} / \mathrm{mL})$, as determined by the CCK-8 method. (C) A subcutaneous tumor model in nude mice was used to detect the inhibition of TP cell proliferation in vivo and confirmed the inhibitory effect of TP on tumor cell proliferation. ** represents $\mathrm{P}<0.01$. TP, triptolide; NC, normal control; DMSO, dimethyl sulfoxide; CCK-8, Cell Counting Kit-8; IC ${ }_{50}$, halfmaximal inhibitory concentration.

passed, until day 7 , indicating that TP has a long-lasting drug effect (Figure 1A,1B).

In our in vivo experiment, the nude mice in the experimental group were injected intraperitoneally with $0.5 \mathrm{mg} / \mathrm{kg}$ TP every other day following successful tumor formation, and the mice in the control group were injected with an equal volume of DMSO. The tumor volume was measured at 4-day intervals. The nude mice were sacrificed on day 28 , and the tumor tissues were collected. The tumors in the TP treatment group were significantly smaller than those in the control group (1 nude mouse in each group died before day 28). The results are shown in Figure 1C.

\section{TP inbibits glioma cell migration and invasion}

We chose 3 observation time points ( 0,12 , and 24 hours) to observe the migration ability of U87 cells, and found that TP significantly inhibited cell migration. As U251 cells often migrate slowly, the observation time was extended to 48 hours. After 48 hours, the cell migration ability of
TP-treated cells was significantly inhibited compared to that of the control cells (Figure 2A-2D). Further, the Transwell invasion experiments showed that TP could also significantly inhibit the invasion ability of U87 and U251 cells in vitro (Figure 3).

\section{$T P$ reverses the EMT process of glioma cells in vitro and in vivo}

In the experimental group, cells were stimulated with TP for 72 hours (concentration: U87: $20 \mathrm{ng} / \mathrm{mL}$; U251: $50 \mathrm{ng} / \mathrm{mL}$ ), and the same amount of DMSO was added to the control group for 72 hours. Proteins were extracted from the U87 and U251 experimental groups and the control group. The western blot results showed that the mesenchymal protein markers $\mathrm{N}$-cadherin, Vimentin, and $\beta$-catenin, together with regulatory factors such as Slug, Snail, and ZEB1, were all downregulated in the TP-treated group, whereas the expression of E-cadherin, an epithelial protein marker, was significantly upregulated. These 

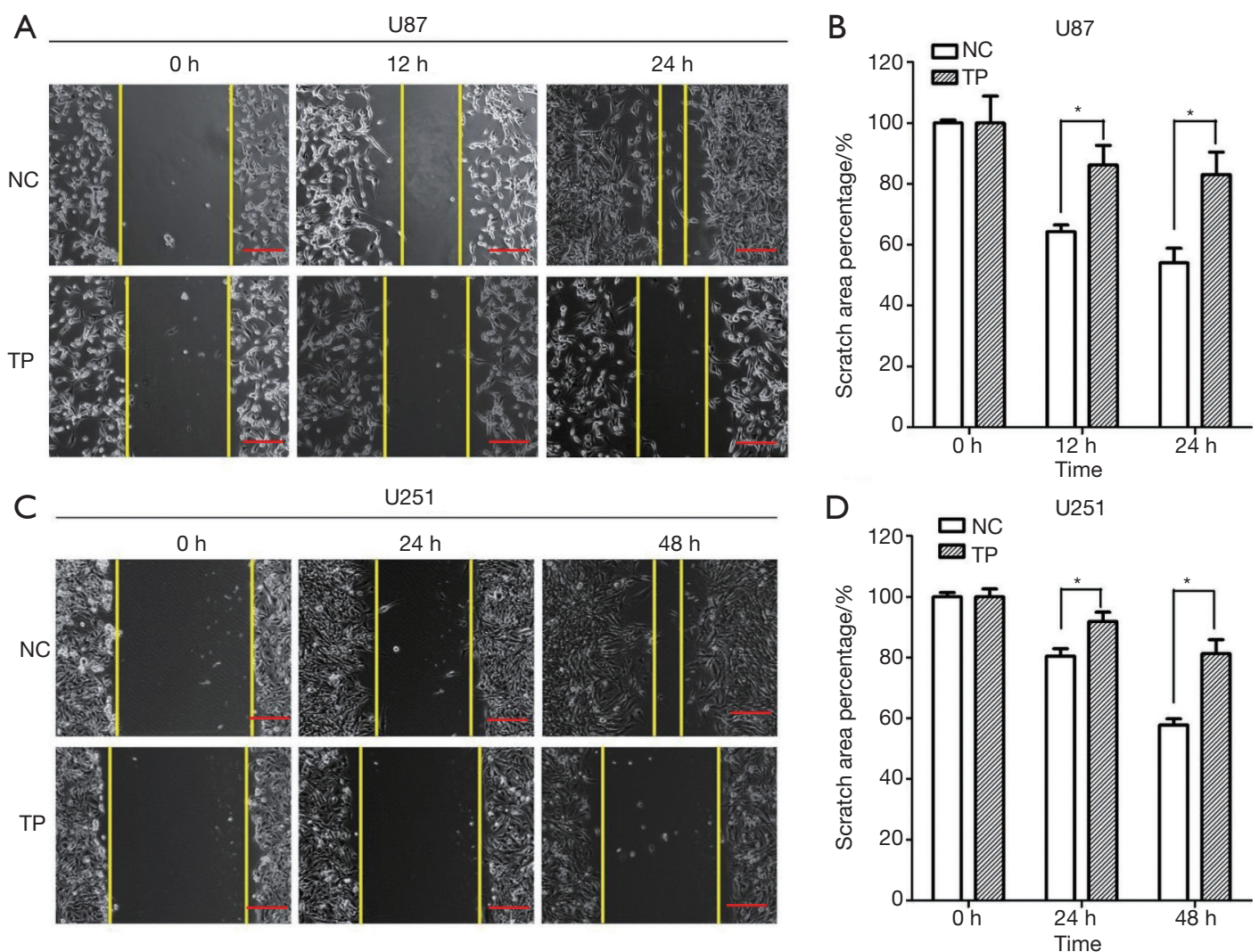

Figure 2 Effect of TP on the migration ability of glioma cells in vitro, as determined by cell scratch test. (A,B) TP significantly inhibits the migration ability of U87 cells. (C,D) TP significantly inhibits the migration ability of U251 cells. Scale bar $=50 \mu \mathrm{m}$. * represents $\mathrm{P}<0.05$. NC, normal control; TP, triptolide.
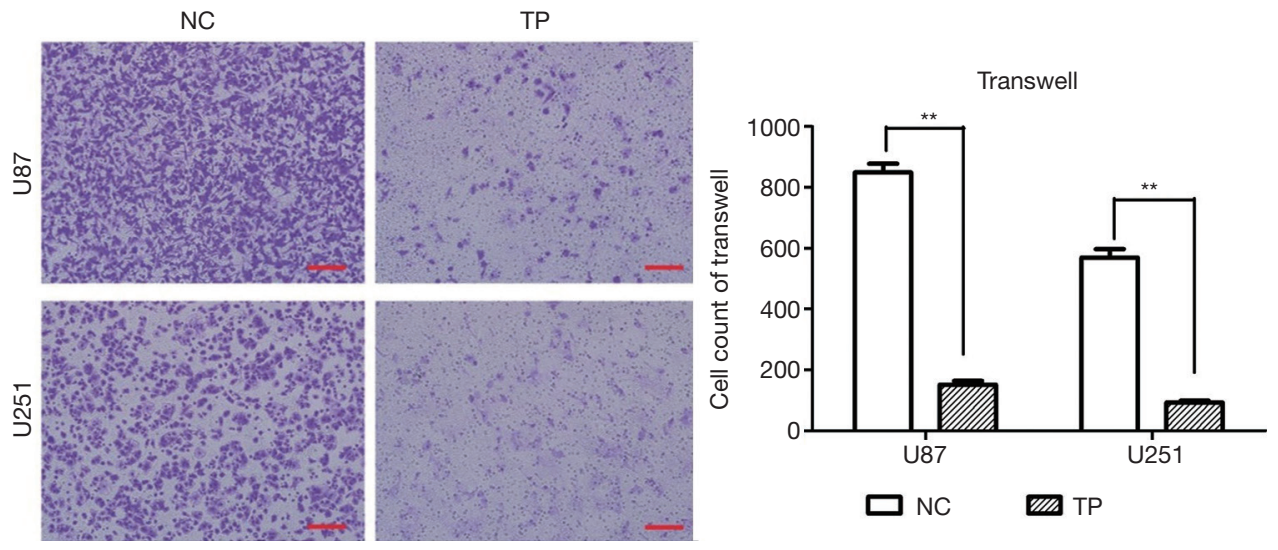

Figure 3 Effect of TP on the invasion ability of glioma cells in vitro, as determined by Transwell invasion assay. (A) TP significantly inhibits the invasion of U87 and U251 cells. Staining method: $0.1 \%$ crystal violet solution. Scale bar $=100 \mu \mathrm{m}$. (B) Statistical data of the invasion experiments on $\mathrm{U} 87$ and $\mathrm{U} 251$ cells stimulated with TP. ** represents $\mathrm{P}<0.01$. NC, normal control; TP, triptolide. 

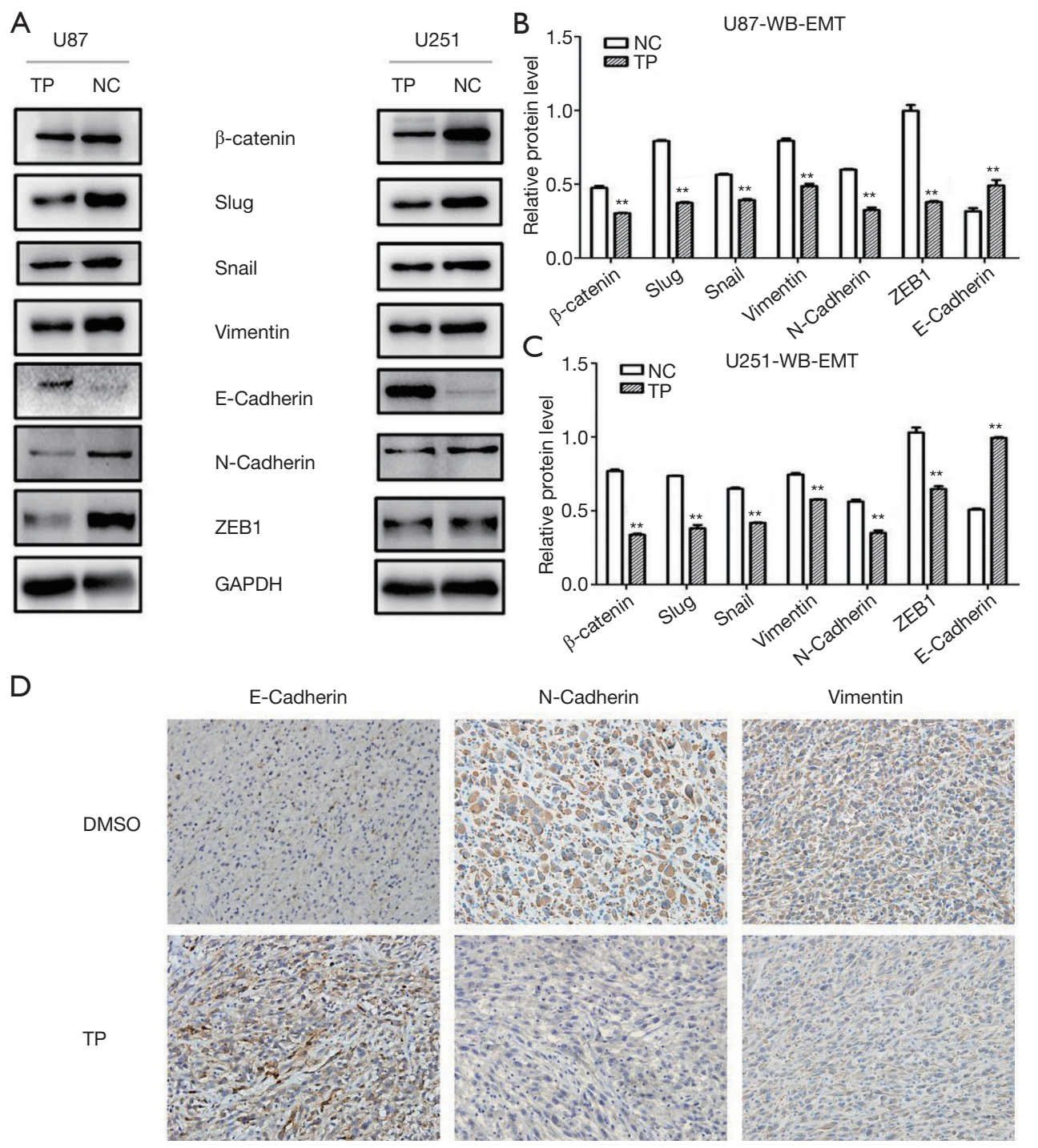

Figure 4 Effects of TP on the EMT process in glioma cells in vitro and in vivo. (A) Western blot results show that TP significantly upregulates the expression of the epithelial marker E-cadherin while downregulating that of the mesenchymal markers $\mathrm{N}$-cadherin, Vimentin, ZEB1, Snail, and Slug in U87 and U251 cells. (B,C) Statistical data from western blot experiments to detect the expression of EMT protein markers in TP-treated U87 cells and U251 cells. (D) Immunohistochemical results confirm that the expression of E-cadherin is upregulated in tumor tissues, while that of $\mathrm{N}$-cadherin and Vimentin is downregulated $(\times 400)$. ${ }^{* *}$ represents $\mathrm{P}<0.01$. TP, triptolide; $\mathrm{NC}$, normal control; EMT, epithelial-mesenchymal transition; DMSO, dimethyl sulfoxide.

results indicated that $\mathrm{TP}$ could inhibit the EMT process (Figure 4A-4C).

In vivo, IHC experiments were performed on subcutaneous tumor tissues from nude mice. Consistent with the results of western blot in vitro, in the tissues of TP-treated mice, the expression of the epithelial marker E-cadherin was significantly upregulated, while the expression of the mesenchymal markers $\mathrm{N}$-cadherin and
Vimentin was significantly downregulated, thus confirming that TP also has a reversal effect on the EMT process in vivo (Figure $4 D$ ).

\section{TP promotes autophagy enhancement of glioma cells}

We found that TP could promote autophagy in glioma cell lines in the early stage (6 hours); however, after 
A

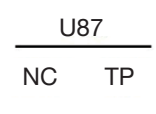

Beclin-1

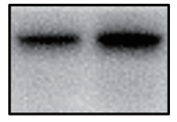

$\operatorname{Atg} 7$
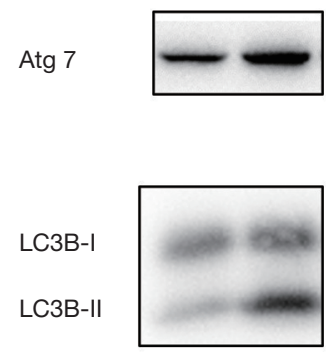

GAPDH

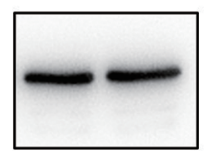

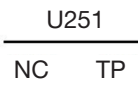
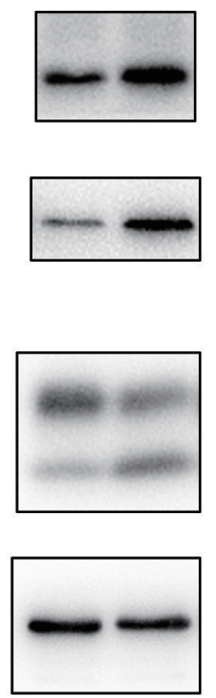

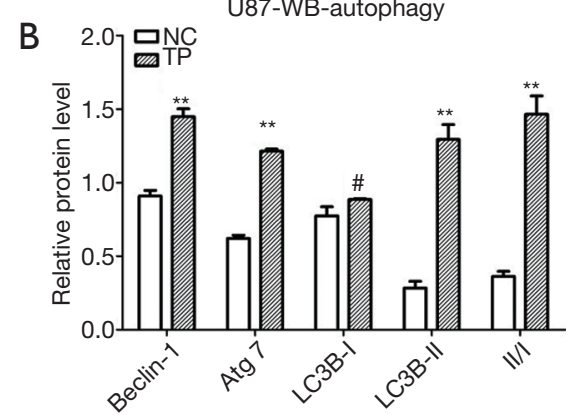

U251-WB-autophagy

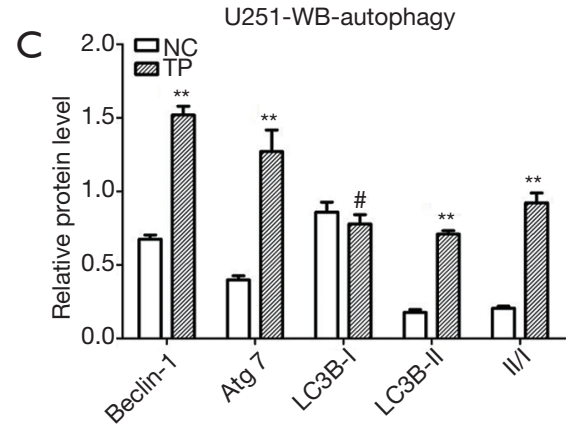

Figure 5 Effects of TP on autophagy in glioma cells in vitro. (A) Western blot results show that TP significantly upregulates Beclin-1 and Atg 7 expression, and increases the LC3B II/I ratio in U87 and U251 cells. Statistical data from western blot experiments to detect the expression of autophagy protein markers in TP-treated (B) U87 cells and (C) U251 cells. ** represents $\mathrm{P}<0.01$; ${ }^{*}$ represents $\mathrm{P}>0.05$. NC normal control; TP, triptolide.

stable stimulation of U87 and U251 cells with TP for 48 hours, the changes in autophagy were more obvious. The expression levels of the autophagy markers Beclin-1 and Atg-7 were significantly elevated; conversion from LC3B I to II was significantly increased; and the LC3B II/ I ratio also showed an increase (Figure 5). After transfection of the pEGFP-C2-LC3B plasmid into glioma cells, the effect of TP on autophagy during the early stage (6 hours) was detected by cell immunofluorescence assay. Under the high-power field of the laser confocal microscope, both U87 cells (Figure 6A) and U251 cells (Figure 6B) exhibited a significant increase in the number of intracytoplasmic autophagosomes (Figure 6C), again showing that TP could promote the autophagy of glioma cells.

\section{Discussion}

As a traditional Chinese medicine, Tripterygium wilfordii Hook F, also known as thunder god vine, has a variety of pharmacological effects, such as detoxifying and dispersing knots, removing blood stasis and promoting blood circulation, eliminating pathogenic factors, and immunosuppression (22). TP, a diterpenoid triepoxide, was first isolated and characterized from Tripterygium wilfordii Hook F (23), in which it is the main active monomer component. Numerous studies have shown that TP is a broad-spectrum tumor inhibitor, which suppresses around 60 types of tumor cells, including melanoma, breast cancer, gastric cancer, colorectal cancer, hepatocellular carcinoma, bile duct carcinoma, lung cancer, pancreatic cancer, prostate cancer, bladder cancer, epidermoid carcinoma, granulocyte leukemia, T-cell lymphoma, and central nervous system tumors (24-26).

In this study, the CCK-8 method was used to detect the effect of TP on the proliferation of the glioma cell lines U87 and U251. The results showed that TP had a significant dose- and time-dependent inhibitory effect on U87 and U251 cells. This part of the experiment confirmed that TP also has an anti-proliferation effect on glioma cells, which is consistent with the literature (27). The subcutaneous xenograft experiment in nude mice further confirmed the inhibitory effect of TP in vivo.

Next, by performing scratch and Transwell invasion assays, we found that TP significantly inhibited the 

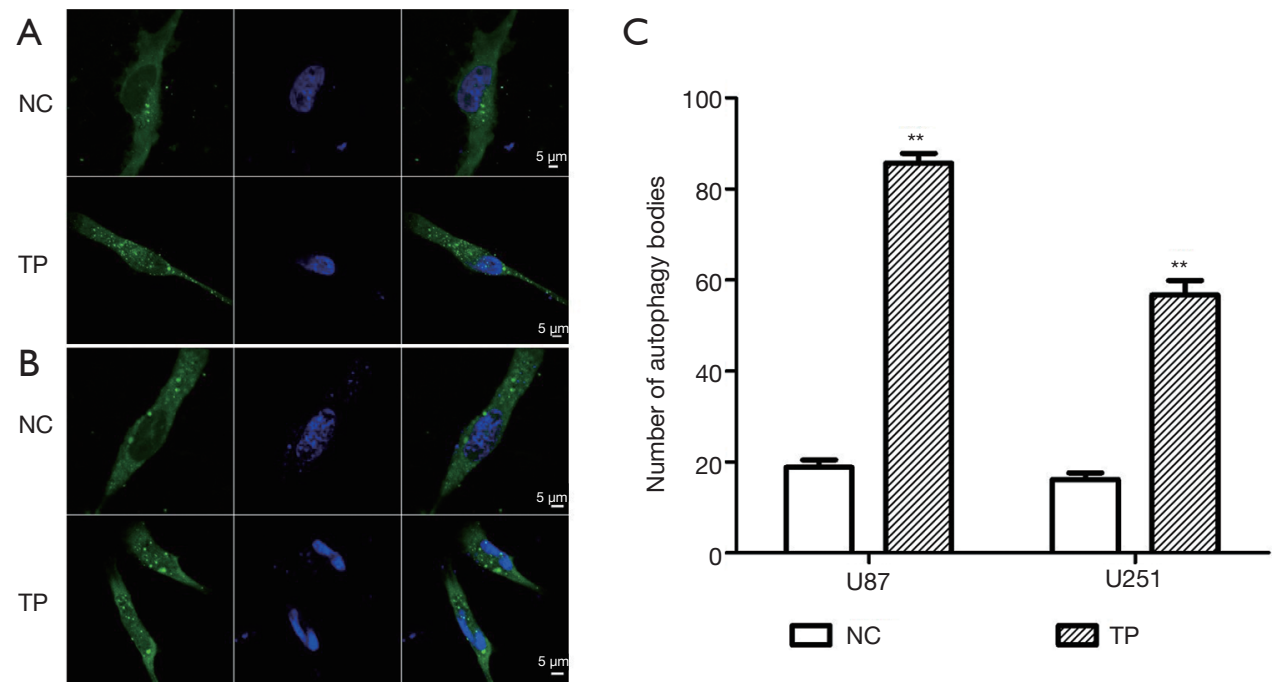

Figure 6 Cellular immunofluorescence detection of autophagic changes in glioma cells under stimulation with TP. (A) Cellular immunofluorescence results show that the number of autophagosomes increased significantly after TP was applied to U87 cells. (B) Cellular immunofluorescence results show that the number of autophagosomes increased significantly after TP was applied to U251 cells. (C) Statistical data of the number of autophagosomes after TP stimulation of U87 and U251 cells. ** represents P<0.01. NC normal control; TP, triptolide.

migration and invasion ability of U87 and U251 cells. Subsequent western blot experiments showed that TP reversed the process of EMT in glioma cells, as evidenced by the upregulation of E-cadherin and the downregulation of N-cadherin, Vimentin, ZEB1, Snail, and Slug.

As we know, based on both tumor morphology and molecular alterations, the WHO 2016 classification system other than the previous one integrated tumor morphology, $1 \mathrm{p} 19 \mathrm{q}$ co-deletion status and IDH mutation for adult diffuse glioma. This is a profound platform that can be utilized to analyze the current and future trends in the diagnosis and prognosis of glioma from molecular biology to proteomics. Recent studies disclosed several signal pathways that closely related to the proliferation and metastasis of glioma, such as IGF-1R/AKT, Notch, mTOR, Wnt and EMT (28-32). EMT is an indispensable part of the normal development process of the human body, and it also plays a crucial role in tumor progression and metastasis. As a biological process, EMT refers to the transformation from immobile epithelial cells to mobile mesenchymal cells whose phenotype is becoming detached and invasive. The mechanism of EMT regulating the invasion and metastasis of glioma is complicated. A variety of transcription factors (such as Snail, Slug, Twist and Zeb 1/2), upstream regulators of EMT induction (such as IGF-1R, c-MET and the CXCR4 receptor) and several key signal pathways (such as PI3K/ AKT and ERK) are involved in the main mechanism (33). Therefore, inhibiting the activation of EMT and epithelialmesenchymal plasticity has become an effective method in the clinical treatment of tumors (34).

Drug resistance, together with radiotherapy resistance, are closely related with tumor recurrence. Drug resistance to $\mathrm{TMZ}$, the first-line chemotherapy drug for glioma, is becoming increasingly prominent, and its mechanism of resistance is still unclear. Researches nowadays indicated that O6-methylguanine-DNA methyltransferase (MGMT) can be the main mechanism of TMZ resistance, while mismatch repair (MMR) and base excision repair (BER) also contribute to it. Emerging evidence also identified that GBM stem cells (GSCs) are tightly connected with drug resistance and tumor recurrence due to self-renewal and invasion ability (3). Once exposed to radiation or TMZ treatment, autophagy is highly triggered and glioma cells could survive by degrading unnecessary components (35). Other studies have found that long-term use of TMZ could induce autophagy and then lead to changes in the morphology of glioma cells and promotes the process of EMT, which indicated another possible chemoresistance mechanism of GBM (36). Snail and Slug, the key molecules involved in EMT, were found being regulated significantly 
upon autophagy stimulation (37). To sum up, EMT also plays a fundamental role in driving drug resistance. Interestingly, its role in cancer drug resistance was not that clear until remarkable similarities between cells undergoing EMT and CSCs (cancer stem cells) were found gradually. It is known that CSCs are extremely hard to eradicate because of its resistance to most of current chemotherapy regimens (38). When molecular switches and key signaling pathways in EMT were better understood, scientists had paid more attention on developing small molecules and miRNAs that reverse EMT phenotype and ultimately enhance the chemo-sensitivity of drug-resistant cancer cells. TP can reverse the EMT process of glioma, which may partially solve the drug-resistance problem facing TMZ. The combination of these two drugs may have the effect of increasing sensitivity to TMZ.

The mechanism by which TP reverses the EMT process is still unclear. Notably, TP can induce autophagy enhancement in a variety of tumors $(18,39,40)$. It is also known that autophagy has a two-sided effect on tumors. On the one hand, it can recover nutrients and aging organelles so that energy can be recovered and utilized, and as such, is conducive to the survival of tumor cells; on the other hand, it inhibits tumor cell growth, activates the apoptotic signaling pathway, and leads to autophagic cell death (41). The results of our western blot and cellular immunofluorescence experiments show that after treatment with TP, the LC3 II/I ratio of U87 and U251 glioma cells was characteristically elevated, indicating the transformation of cytoplasmic type LC3 (LC3-I) into autophagosome membrane type LC3 (LC3-II) after enzymolysis results in the promotion of autophagosome formation and autophagic enhancement. As we know, Beclin-1 plays an important role in membrane isolation and vesicle closure of autophagosomes. It can interact with $\mathrm{P} 53$ protein, the Bcl2 protein family, and other proteins to affect autophagy. ATG-7 is one of the proteins involved in autophagy. Our results revealed that the expression levels of Beclin-1 and ATG-7 in TP-treated cells were upregulated, further supporting that TP induces the enhancement of autophagy in glioma cells.

As mentioned above, the key marker of the EMT process is decreased E-cadherin expression. EMT factors such as Snail, Slug, ZEB1, Twist1, Twist2, and SIP1 can bind to the E-cadherin promoter's E-box (enhancer box which located within the short-range transcription regulatory regions of the E-cadherin gene) to inhibit the transcription of
E-cadherin. Our experiment showed that after stimulation of glioma cells with TP, the expression of ZEB1, Snail, and Slug was downregulated. This suggests that the overexpression of E-cadherin induced by TP was possibly caused by the downregulation of upstream factors such as ZEB1, Snail, and Slug, which in turn reduced the inhibitory effect on E-cadherin and thus reversed EMT progression. There is evidence that the activation and enhancement of autophagy can reverse the EMT phenotype of tumor cells, thereby inhibiting the occurrence of EMT $(42,43)$. Studies have found that in glioma cells, the enhancement of autophagy is induced by stress or nutritional deficiency, while EMT is inhibited through the classical mTOR pathway, resulting in reduced cell migration and weakened invasion abilities (37). Therefore, we can infer that TP can induce autophagy enhancement in U87 and U251 glioma cells, and alter the expression of E-cadherin and Vimentin through ZEB1, Snail, and other pathways mediated by autophagy, thus inhibiting the EMT process.

In summary, we have investigated the effects of $\mathrm{TP}$ on migration, invasion, and EMT progression in glioma cells. We have preliminarily studied and identified that, mechanistically, TP induces autophagy enhancement, thus reversing EMT. This mechanism of EMT reversal may become an entry point for avoiding acquired drug resistance to TMZ. The addition of TP may increase TMZ sensitivity and enhance antitumor efficacy; however, first, the specific pathway of TP-induced autophagy enhancement in tumor cells needs to be further elucidated.

\section{Acknowledgments}

Funding: This work was supported by the Science and Technology Plan of Health Commission of Jiangxi Province (grant no. 20203201 and 20203200), the Science and Technology Research Project of the Education Department of Jiangxi Province (grant no. GJJ190063), and the Natural Science Foundation of Jiangxi Province (grant no. 20202BAB216030).

\section{Footnote}

Reporting Checklist: The authors have completed the ARRIVE reporting checklist. Available at https://dx.doi. org/10.21037/atm-21-2944

Data Sharing Statement: Available at https://dx.doi. 
org/10.21037/atm-21-2944

Conflicts of Interest: All authors have completed the ICMJE uniform disclosure form (available at https://dx.doi. org/10.21037/atm-21-2944). The authors have no conflicts of interest to declare.

Ethical Statement: The authors are accountable for all aspects of the work in ensuring that questions related to the accuracy or integrity of any part of the work are appropriately investigated and resolved. This study was performed under a project license (No. 2020117) granted by the ethics board of the First Affiliated Hospital of Nanchang University and was carried out in strict accordance with the recommendations in the Guide for the Care and Use of Laboratory Animals of the National Institutes of Health. The study was consistent with the principles used to relieve the pains of animals as well as using the least number of animals as possible.

Open Access Statement: This is an Open Access article distributed in accordance with the Creative Commons Attribution-NonCommercial-NoDerivs 4.0 International License (CC BY-NC-ND 4.0), which permits the noncommercial replication and distribution of the article with the strict proviso that no changes or edits are made and the original work is properly cited (including links to both the formal publication through the relevant DOI and the license). See: https://creativecommons.org/licenses/by-nc-nd/4.0/.

\section{References}

1. Louis DN, Ohgaki H, Wiestler OD, et al. The 2007 WHO classification of tumours of the central nervous system. Acta Neuropathol 2007;114:97-109.

2. Stupp R, Mason WP, van den Bent MJ, et al. Radiotherapy plus concomitant and adjuvant temozolomide for glioblastoma. N Engl J Med 2005;352:987-96.

3. Jiapaer S, Furuta T, Tanaka S, et al. Potential Strategies Overcoming the Temozolomide Resistance for Glioblastoma. Neurol Med Chir (Tokyo) 2018;58:405-21.

4. Yi GZ, Huang G, Guo M, et al. Acquired temozolomide resistance in MGMT-deficient glioblastoma cells is associated with regulation of DNA repair by DHC2. Brain 2019;142:2352-66.

5. Ratel D, van der Sanden B, Wion D. Glioma resection and tumor recurrence: back to Semmelweis. Neuro Oncol 2016;18:1688-9.
6. Hsu YY, Jong YJ, Tsai HH, et al. Triptolide increases transcript and protein levels of survival motor neurons in human SMA fibroblasts and improves survival in SMA-like mice. Br J Pharmacol 2012;166:1114-26.

7. Chen BJ. Triptolide, a novel immunosuppressive and anti-inflammatory agent purified from a Chinese herb Tripterygium wilfordii Hook F. Leuk Lymphoma 2001;42:253-65.

8. Brabletz T, Jung A, Reu S, et al. Variable beta-catenin expression in colorectal cancers indicates tumor progression driven by the tumor environment. Proc Natl Acad Sci U S A 2001;98:10356-61.

9. Brabletz T, Jung A, Spaderna S, et al. Opinion: migrating cancer stem cells - an integrated concept of malignant tumour progression. Nat Rev Cancer 2005;5:744-9.

10. Han SP, Kim JH, Han ME, et al. SNAI1 is involved in the proliferation and migration of glioblastoma cells. Cell Mol Neurobiol 2011;31:489-96.

11. Kahlert UD, Maciaczyk D, Doostkam S, et al. Activation of canonical WNT/ $\beta$-catenin signaling enhances in vitro motility of glioblastoma cells by activation of ZEB1 and other activators of epithelial-to-mesenchymal transition. Cancer Lett 2012;325:42-53.

12. Nomura A, Majumder K, Giri B, et al. Inhibition of NFkappa B pathway leads to deregulation of epithelialmesenchymal transition and neural invasion in pancreatic cancer. Lab Invest 2016;96:1268-78.

13. Liu L, Salnikov AV, Bauer N, et al. Triptolide reverses hypoxia-induced epithelial-mesenchymal transition and stem-like features in pancreatic cancer by NF- $\mathrm{\kappa B}$ downregulation. Int J Cancer 2014;134:2489-503.

14. Li F, Cui H, Jin X, et al. Triptolide inhibits epithelial-mesenchymal transition and induces apoptosis in gefitinib-resistant lung cancer cells. Oncol Rep 2020;43:1569-79.

15. Reno TA, Kim JY, Raz DJ. Triptolide Inhibits Lung Cancer Cell Migration, Invasion, and Metastasis. Ann Thorac Surg 2015;100:1817-24; discussion 1824-5.

16. Acikgoz E, Tatar C, Oktem G. Triptolide inhibits CD133+ /CD44+ colon cancer stem cell growth and migration through triggering apoptosis and represses epithelialmesenchymal transition via downregulating expressions of snail, slug, and twist. J Cell Biochem 2020;121:3313-24.

17. Boya P, Reggiori F, Codogno P. Emerging regulation and functions of autophagy. Nat Cell Biol 2013;15:713-20.

18. Liu X, Zhao P, Wang X, et al. Triptolide Induces Glioma Cell Autophagy and Apoptosis via Upregulating the ROS/ JNK and Downregulating the Akt/mTOR Signaling 
Pathways. Front Oncol 2019;9:387.

19. Kroemer G, Mariño G, Levine B. Autophagy and the integrated stress response. Mol Cell 2010;40:280-93.

20. Lazova R, Camp RL, Klump V, et al. Punctate LC3B expression is a common feature of solid tumors and associated with proliferation, metastasis, and poor outcome. Clin Cancer Res 2012;18:370-9.

21. Avivar-Valderas A, Bobrovnikova-Marjon E, Alan Diehl J, et al. Regulation of autophagy during ECM detachment is linked to a selective inhibition of mTORC1 by PERK. Oncogene 2013;32:4932-40.

22. Chen SR, Dai Y, Zhao J, et al. A Mechanistic Overview of Triptolide and Celastrol, Natural Products from Tripterygium wilfordii Hook F. Front Pharmacol 2018;9:104.

23. Kupchan SM, Court WA, Dailey RG Jr, et al. Triptolide and tripdiolide, novel antileukemic diterpenoid triepoxides from Tripterygium wilfordii. J Am Chem Soc 1972;94:7194-5.

24. Noel P, Von Hoff DD, Saluja AK, et al. Triptolide and Its Derivatives as Cancer Therapies. Trends Pharmacol Sci 2019;40:327-41.

25. Jiang W, Chen M, Xiao C, et al. Triptolide Suppresses Growth of Breast Cancer by Targeting HMGB1 in Vitro and in Vivo. Biol Pharm Bull 2019;42:892-9.

26. Noel P, Hussein S, Ng S, et al. Triptolide targets superenhancer networks in pancreatic cancer cells and cancerassociated fibroblasts. Oncogenesis 2020;9:100.

27. Zhang $\mathrm{H}, \mathrm{Zhu} \mathrm{W}, \mathrm{Su} \mathrm{X}$, et al. Triptolide inhibits proliferation and invasion of malignant glioma cells. J Neurooncol 2012;109:53-62.

28. Yang Q, Deng L, Li J, et al. NR5A2 Promotes Cell Growth and Resistance to Temozolomide Through Regulating Notch Signal Pathway in Glioma. Onco Targets Ther 2020;13:10231-44.

29. Yu M, Yu S, Gong W, et al. Knockdown of linc01023 restrains glioma proliferation, migration and invasion by regulating IGF-1R/AKT pathway. J Cancer 2019;10:2961-8.

30. Shen B, Sun D. Natural Diterpenoid Isoferritin A (IsoA) Inhibits Glioma Cell Growth and Metastasis via Regulating of TGF $\beta$-Induced EMT Signal Pathway. Med Sci Monit 2018;24:3815-23.

31. Gao C, Liang C, Nie Z, et al. Alkannin inhibits growth and invasion of glioma cells C6 through IQGAP/mTOR signal pathway. Int J Clin Exp Med 2015;8:5287-94.

32. Wu S, Luo C, Li F, et al. Silencing expression of PHF14 in glioblastoma promotes apoptosis, mitigates proliferation and invasiveness via Wnt signal pathway. Cancer Cell Int 2019;19:314.

33. Liu CA, Chang CY, Hsueh KW, et al. Migration/Invasion of Malignant Gliomas and Implications for Therapeutic Treatment. Int J Mol Sci 2018;19:1115.

34. Nantajit D, Lin D, Li JJ. The network of epithelialmesenchymal transition: potential new targets for tumor resistance. J Cancer Res Clin Oncol 2015;141:1697-713.

35. Kanzawa T, Germano IM, Komata T, et al. Role of autophagy in temozolomide-induced cytotoxicity for malignant glioma cells. Cell Death Differ 2004;11:448-57.

36. Lu Y, Xiao L, Liu Y, et al. MIR517C inhibits autophagy and the epithelial-to-mesenchymal (-like) transition phenotype in human glioblastoma through KPNA2dependent disruption of TP53 nuclear translocation. Autophagy 2015;11:2213-32.

37. Catalano M, D'Alessandro G, Lepore F, et al. Autophagy induction impairs migration and invasion by reversing EMT in glioblastoma cells. Mol Oncol 2015;9:1612-25.

38. Zhao J. Cancer stem cells and chemoresistance: The smartest survives the raid. Pharmacol Ther 2016;160:145-58.

39. Gao H, Zhang Y, Dong L, et al. Triptolide induces autophagy and apoptosis through ERK activation in human breast cancer MCF-7 cells. Exp Ther Med 2018;15:3413-9.

40. Qin G, Li P, Xue Z. Triptolide induces protective autophagy and apoptosis in human cervical cancer cells by downregulating Akt/mTOR activation. Oncol Lett 2018;16:3929-34.

41. Lv Q, Wang W, Xue J, et al. DEDD interacts with PI3KC3 to activate autophagy and attenuate epithelialmesenchymal transition in human breast cancer. Cancer Res 2012;72:3238-50.

42. Li G, Li CX, Xia M, et al. Enhanced epithelial-tomesenchymal transition associated with lysosome dysfunction in podocytes: role of p62/Sequestosome 1 as a signaling hub. Cell Physiol Biochem 2015;35:1773-86.

43. Chen HT, Liu H, Mao MJ, et al. Crosstalk between autophagy and epithelial-mesenchymal transition and its application in cancer therapy. Mol Cancer 2019;18:101.

(English Language Editors: M. Bucci and J. Reynolds)

Cite this article as: Lai M, Liu L, Zhu L, Feng W, Luo J, Liu Y, Deng S. Triptolide reverses epithelial-mesenchymal transition in glioma cells via inducing autophagy. Ann Transl Med 2021;9(16):1304. doi: 10.21037/atm-21-2944 